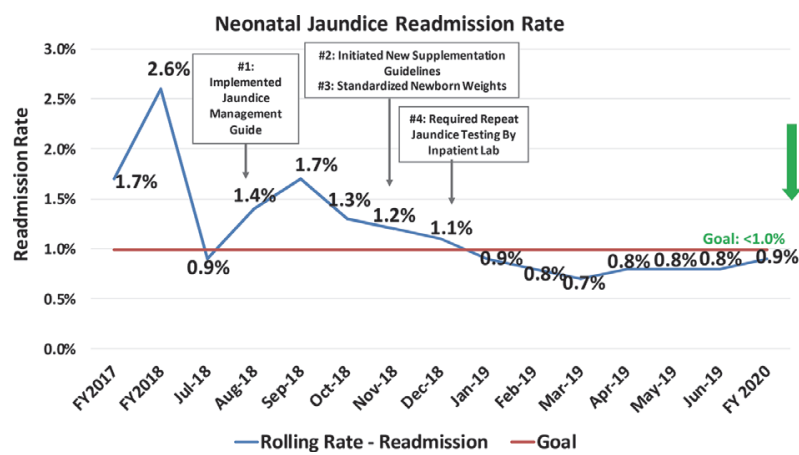

Abstract 19 Figure 3

than accepting lab values from other facilities which were often found to be different (figure 2).

Results Overall, the rolling percentage of neonatal jaundice readmissions for BSWMCW decreased from $2.6 \%$ to $0.8 \%$, meeting the goal of less than $1 \%$. This was sustained through fiscal year 2019 (figure 3).

Conclusions Neonatal Jaundice readmission rates can be affected through standardizing jaundice management protocols. In changing the timing of neonatal weights, this improved neonatal care, even for those without jaundice.

\section{OPTIMIZING INPATIENT SITUATION AWARENESS TO RECOGNIZE AND MITIGATE CLINICAL DETERIORATION IN HOSPITALIZED CHILDREN}

Tina Sosa, Maya Dewan, Michelle Coleman, Brandy Seger, Jackie Hausfeld, Richard Falcone, Patrick Brady, Jeffrey Simmons, Christine White, Mary Sitterding. Cincinnati Children's Hospital Medical Center, USA

\subsection{6/bmjoq-2020-IHI.20}

Background Interventions to improve care team situation awareness (SA) are associated with reduced rates of unrecognized clinical deterioration in hospitalized children. Recent safety events at our institution revealed common etiologic themes, including 1) inadequate SA for patients demonstrating signs of deterioration, and 2) lack of a shared mental model due to inadequate psychological safety and communication.

Objectives We aimed to decrease emergency transfers (ETs) to the intensive care unit (ICU) by $50 \%$ over 10 months.

Methods An interprofessional team of physicians, nurses, respiratory therapists, and families convened to apply innovation to the original SA model for clinical deterioration by addressing emerging corruptors to SA, communication inadequacies, and evolving technology in our inpatient system. Key drivers included establishing a shared mental model, psychologically safe escalation, and efficient and effective SA tools (figure 1). Novel interventions including the intentional inclusion of families and diverse care team roles in huddles, a mental model checklist, door signage, and an electronic health record SA navigator were evaluated via a time series analysis. Sequential inpatient-wide testing of the SA model allowed for iteration and consensus building across care teams and families via qualitative data collection and review. The primary outcome measure was ETs, defined as any ICU transfer where the patient

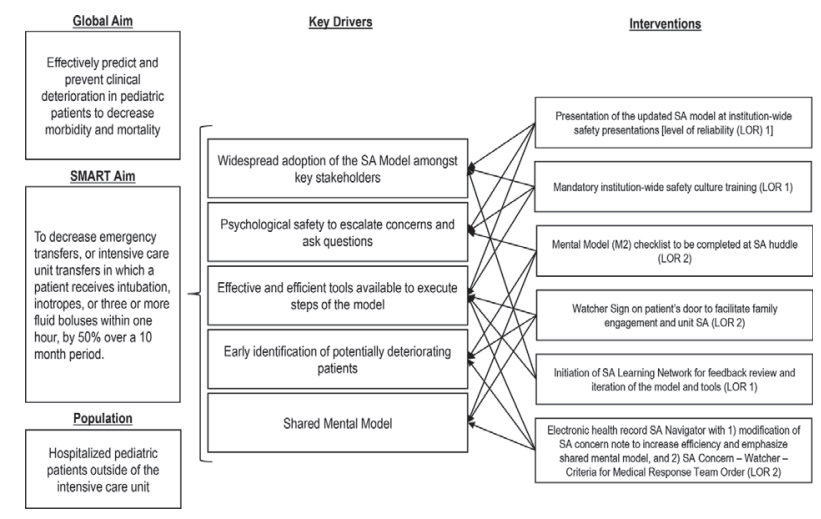

Abstract 20 Figure 1 Key driver diagram for the SA Model for clinical deterioration

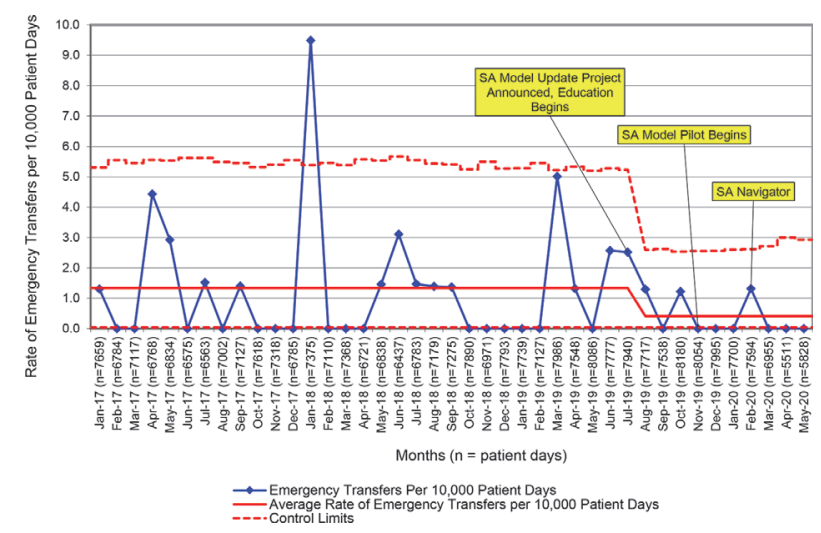

Abstract 20 Figure 2 Outcome measure: emergency transfers. Statistical process control U-chart of the rate of emergenc transfers per 10,000 patient days. Established rules for shewhart control were utilized to determine if observed changes were due to special causes variation
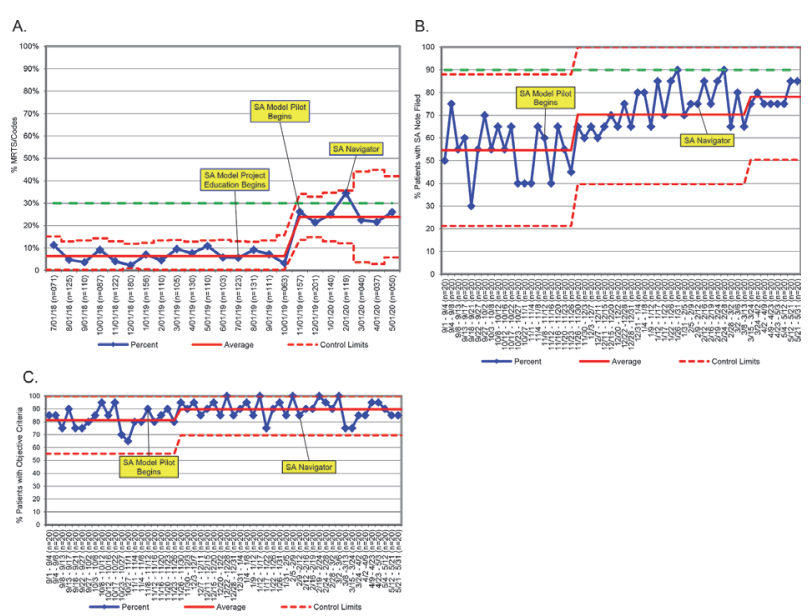

Abstract 20 Figure 3 Process measures. Statistical process control Pcharts: A) Percent of patients designated as watchers at least one hour prior to medical response team or code activation. B) Percent of watcher patients who have an SA note filed within two hours of watcher status initiation. C) Percent of watcher patients who have objective medical response team activation criteria. Established rules for Shewhart control charts were utilized to determine If observed changes were due to special cause variation 
received intubation, inotropes, or $\geq 3$ fluid boluses within one hour of transfer.

Results The average rate of ETs per 10,000 patient days decreased from 1.57 to 0.49 during the study period (figure 2). This coincided with special cause improvement in all process measures, including earlier recognition of potentially deteriorating patients and increased exemplary utilization of SA tools (figure 3).

Conclusions An innovative, proactive, and reliable process to predict, prevent, and respond to clinical deterioration was associated with a nearly $70 \%$ reduction in ETs. Importantly, ETs are associated with increased hospital length of stay and mortality.

\section{IMPLEMENTING TEAM BASED CARE TO IMPROVE CERVICAL CANCER SCREENING RATE IN COMMUNITY BASED RESIDENT RUN CLINIC}

Momena Sohail, Fatima Fayyaz, Sarah Finlay, Afnan Ammar. Trinity Health, USA

\subsection{6/bmjoq-2020-IHI.21}

Background In 2015, for every 100,000 women, 8 new Cervical Cancer cases were reported and 2 died of cancer. The introduction of effective screening has led to a steady decrease in Invasive Cervical Cancer incidence and mortality in highincome countries however Socioeconomic status and access to health have created a cervical cancer disparity gap. This QI project was undertaken to evaluate and improve cervical cancer screening rates at a community-based resident-run IM clinic.

Objectives Increase screening rate to $50 \%$ by Dec 2018 and sustain increase rates to above $45 \%$ in all months in 2019.

Methods At the start of 2018, physician data reported that screening rates at the clinic were $36 \%$, lower than those of our other clinical practices. A clinical survey was completed to assess patient understanding of cervical cancer screening. The first intervention was sharing individual provider data with the residents. This was a weak intervention and did not improve rates of screening. Our second intervention was a team-based approach and pre-visit planning: identify patients and calling them1-2 days ahead and including PAP in the daily huddle.

Results The original survey showed that out of 124 women, 66 needed screening. Surveys intensified lack of continuity; several listed PCPs were not seeing their own patients, patients unsure of resources, and financial concerns as barriers. The team-based approach showed that more PAPs were being done per week, and was streamlined with staff feedback to minimize disruption to current workflow. With a goal of $50 \%$ by the end of 2018 , we were able to achieve $48.9 \%$ screening rates. The increase was sustained above $45 \%$ in $8 / 12$ months in 2019 (figure 1).

Conclusions Team-based practice is an effective practice in increasing cervical cancer screening to overcome challenges in continuity of care, health access disparity, and resource allocation in a primary care clinic.

\section{IMPLEMENTATION OF A PHARMACIST-DRIVEN ANTIMICROBIAL TIME-OUT FOR MEDICAL-SURGERY SERVICES IN AN ACADEMIC PEDIATRIC HOSPITAL}

Christopher Stang ${ }^{1}$, Jessica Tansmore ${ }^{1}$, Katelyn Parson", Kathryn Nuss ${ }^{1}$, Matthew Sapko ${ }^{1}$ Zachary Thompson", Don Buckingham1, Mahmoud Abdel-Rasoul', Joshua Watson", Preeti Jaggi ${ }^{2}$. 'Nationwide Children's Hospital, United States; ${ }^{2}$ Children's Healthcare of Atlanta, United States

\subsection{6/bmjoq-2020-IHI.22}

Background This quality improvement initiative implemented a pharmacist-driven antimicrobial time-out (ATO) in a large, free-standing pediatric hospital.

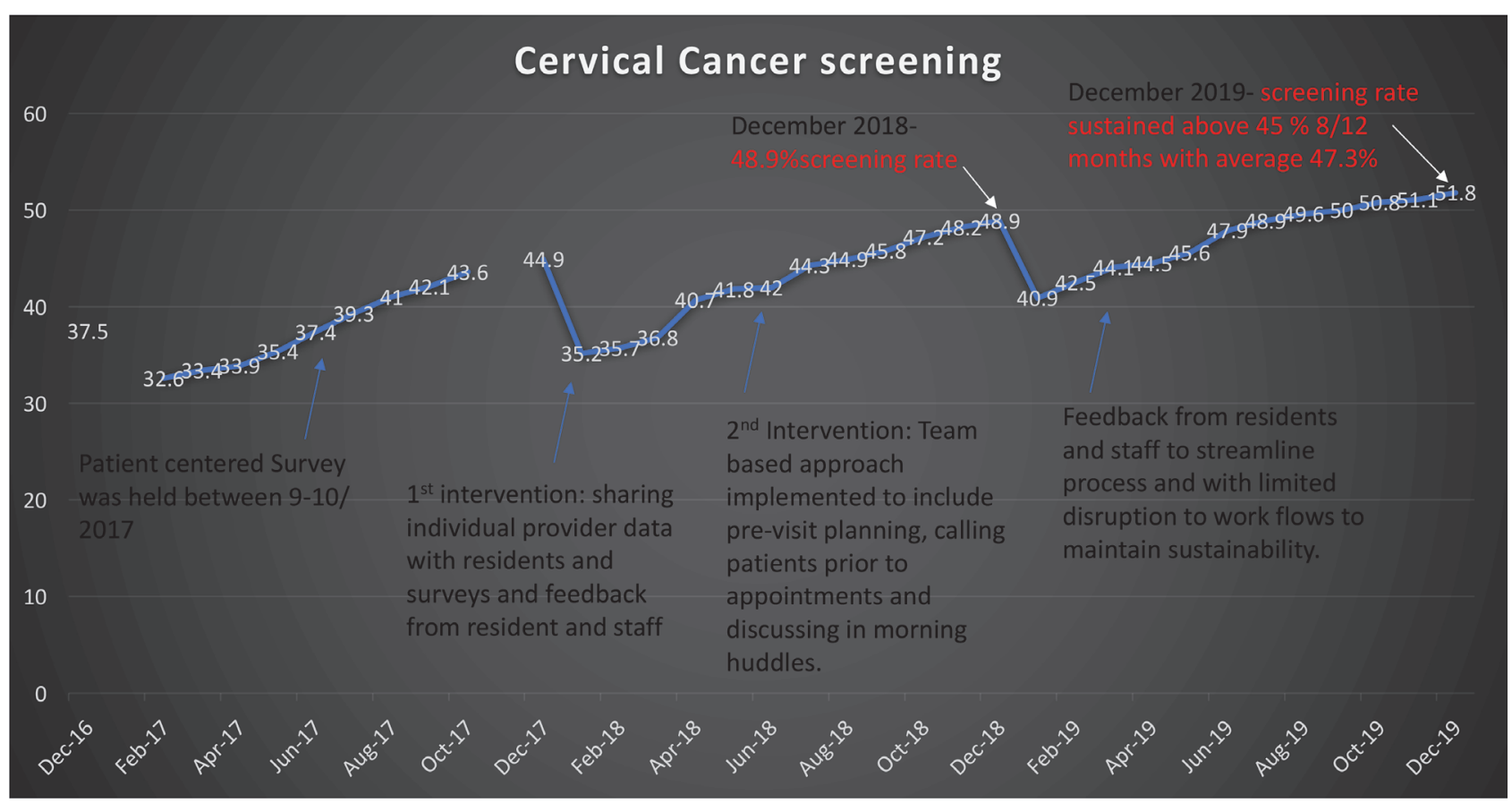

Abstract 21 Figure 1 